\title{
Nonlinear air-coupled thermosonics for fatigue micro-damage detection and localisation
}

\author{
Artur Dyrwal, Michele Meo, Francesco Ciampa * \\ Materials and Structures Centre (MAST), Department of Mechanical Engineering, University of Bath, Claverton Down, BA2 7AY, Bath, UK
}

\section{A R T I C L E I N F O}

\section{Keywords:}

Air-coupled transducers

Thermosonics

Thermography

Nonlinear ultrasounds

Laser vibrometry

\begin{abstract}
A B S T R A C T
Over the years, traditional active infrared thermography has played a pivotal role in ensuring that a component is free of any damage. However, whilst optical thermography is still not sufficiently sensitive to the presence of material micro-flaws, current vibro-thermography requires inspection solutions that are not always feasible, such as the use of coupling materials between the sensing probe and the monitored structure. This paper presents a valid alternative to current thermographic systems by developing and experimentally validating nonlinear aircoupled thermosonics for a contactless, rapid and accurate detection of fatigue micro-cracks in an aero-engine turbine blade. The proposed thermographic method combines the high sensitivity to micro-damage of nonlinear ultrasonic techniques with non-contact air-coupled ultrasonic transducers and thermographic equipment. Narrowband frequency sweeps were performed to identify local damage resonance frequencies in order to generate large vibrational amplitudes at the damage location and compensate for signal losses caused by the high acoustic impedance mismatch between the air and the sample. An infrared camera was then used to acquire the thermal response generated by frictional heat at the crack interfaces. Moreover, an image processing method based on a combination of morphological opening and a Savitzky-Golay smoothing filter was employed to enhance the quality of thermal images affected by anisotropic heating and thermal noise effects. Nonlinear aircoupled thermosonics experiments were validated with laser Doppler vibrometry scan measurements and compared with both flash and pulsed phase thermography. Thermal imaging results showed that the proposed nonlinear air-coupled thermosonics was the only thermographic technique able to detect fatigue micro-cracks, thus demonstrating its potential as an efficient and sensitive inspection tool for micro-damage detection in geometrically complex components.
\end{abstract}

\section{Introduction}

The ongoing rapid advancements in material science and the resultant increasing use of more complex materials and geometries in engineering design have driven the need for innovation and improvement of current non-destructive evaluation (NDE) techniques. Among NDE inspection methods, thermosonics has gained research momentum owing to its versatility, high testing speed, full-field capability and reduced dependence on the operator [1,2]. Thermosonics (also referred to as vibro-thermography or ultrasonic stimulated thermography or sonic IR) is an active infrared (IR) thermographic method for material micro-damage inspection that involves the generation of powerful vibrations in a test piece to cause frictional heating at crack interfaces [3]. These vibrations are produced by an ultrasonic welding horn being pressed against the surface of the part under inspection. However, the coupling between the test specimen and the horn typically results in an uncontrolled generation of frequency components known as "acoustic chaos". Such a condition makes this method highly non-reproducible, thus leading to cracks being undetected if sufficient vibrational energy is not applied at the crack location. In addition, the exposure to high-power excitation generated by the horn may even further degrade the structural integrity of components [4]. Numerous thermographic strategies and approaches have been developed in recent years to improve the reliability of thermosonics. One of the major technological advances was the combination of nonlinear ultrasonic methods using piezoelectric (PZT) transducers and thermography, which gave rise to nonlinear ultrasonic stimulated thermography (NUST) [5-7]. Nonlinear ultrasonic methods have been gaining increasing popularity due to their high sensitivity to micro-damage (e.g. incipient fatigue cracks) over classical linear methods (e.g. C-scan and linear phased array) [8-12].

\footnotetext{
* Corresponding author.

E-mail address: f.ciampa@bath.ac.uk (F. Ciampa).
} 
Indeed, linear elastic wave propagation phenomena such as wave scattering and attenuation induced by micro-defects are generally too small to generate wave distortions, so that micro-flaws tend to remain "invisible". Nonlinear ultrasounds, on the other hand, measure material nonlinear effects such as harmonics and sub-harmonics (i.e. multiples and submultiples) of the driving frequency in order to reveal the presence of surface and sub-surface micro-flaws [13]. These effects are mainly caused by the local vibration of micro-cracks, which produces clapping motion and frictional contact between damage surfaces. Since the generation of higher harmonics indicates the presence of damage, they can be used as a signature for a reliable driving frequency selection. The challenge associated with the use of PZT sensors is, however, the question of whether they are capable of injecting sufficient energy into the specimen to produce heat dissipation through friction. This issue has been recently addressed by the analysis of the local defect resonance (LDR) effect. According to the LDR theory $[14,15,17]$, if the driving frequency matches the damage resonance one, the vibrational amplitude of nonlinear elastic phenomena can be dramatically increased by nearly $20-40 \mathrm{~dB}$. Hence, the acoustic interaction between elastic waves travelling through the specimen and the defect can be maximised, thus reducing the input power to a few $\mathrm{mW}$. This enables the PZT transducers to be reliably used as excitation devices. Furthermore, the LDR effect can push the boundary of NUST even further by supporting the implementation of contactless air-coupled transducers (ACTs), thus making NUST a fully non-contact NDE method. Indeed, for practical applications, it is not always possible or feasible to bond transducers and use water or other liquids as the coupling medium between the sensing probe and the monitored structure. A number of studies in literature have shown the feasibility of using non-contact sensing devices and ACTs in both linear and nonlinear ultrasonic applications [18-23]. The use of ultrasounds with air-coupled transducers is known in literature as air-coupled ultrasounds (ACU). Zalameda et al. [24] at NASA firstly used amplified loudspeakers to excite standing waves on a damaged metallic sample and generate frictional heat at the defect location. However, loudspeakers are only able to transmit input pulses at low frequency ranges (typically from $\sim 20 \mathrm{~Hz}$ to $\sim 10 \mathrm{kHz}$ ), thus limiting the damage detection capabilities to $\mathrm{cm}$-size flaws. To overcome this issue, ACU is proposed in this research, as it allows ultrasonic transmission at higher frequencies to assess material flaws of smaller dimensions. Nevertheless, the use of air as the coupling medium between the transducer and the damaged structure poses major challenges such as high losses in the transmitted signal caused by the severe acoustic impedance mismatch [25]. In this regard, Solodov et al. [26] recently revealed that loudspeakers were able to produce a sound intensity (pressure) of $90 \mathrm{~dB}$ at few $\mathrm{kHz}$ range on a damaged carbon fibre (CFRP) composite panel with "artificial" $\mathrm{cm}$-size flaws (i.e. flat bottom holes). This level of sound intensity combined with the LDR "amplification" of $25 \mathrm{~dB}$ was sufficient to generate standing waves in the sample and frictional contact between damage surfaces, with temperature variations of $30 \mathrm{mK}$ at the defect location. This result opens opportunities for the use of powerful commercial ACTs with thermography that, despite being affected by signal losses in the range of $70-80 \mathrm{~dB}$ due to the acoustic mismatch between air and solid, are capable of providing sound intensities of $\sim 10^{-2} \mathrm{~W} / \mathrm{m}^{2}$ in materials at frequency ranges broader than loudspeakers [19].

The aim of this study is to develop and experimentally validate a fully non-contact thermal imaging technique, here named as nonlinear aircoupled thermosonics (NACT), for the detection and localisation of fatigue micro-flaws in an aircraft engine turbine blade. The application of nonlinear ACU is particularly relevant as both ultrasonic horns and contact PZT transducers require consistent and reliable contact conditions that are cumbersome to achieve due to the small dimensions and complex geometry of the specimen. Three key aspects of the proposed NACT were investigated in this paper: (i) the optimisation process for the nonlinear ACU transmission, (ii) the accurate selection of the LDR frequency using narrowband sweep excitation and (iii) the enhancement of image processing using morphological opening and a Savitzky-Golay

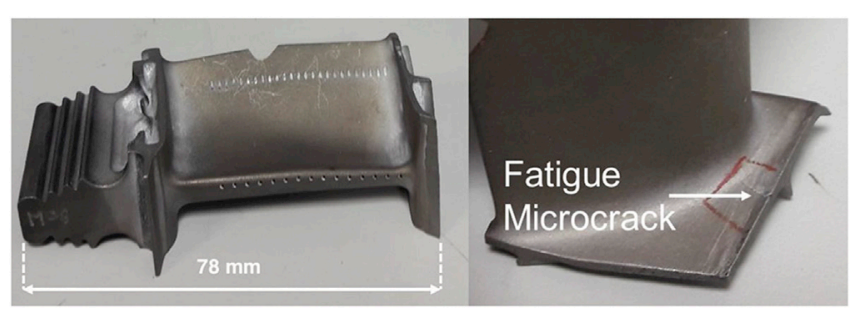

Fig. 1. Inconel aircraft engine turbine blade with a $1.4 \mathrm{~mm}$ fatigue micro-crack in its shroud.

smoothing filter. Moreover, NACT thermal results were validated with two-dimensional (2D) laser Doppler vibrometry scan measurements and compared with traditional thermography. The outline of this research work is as follows: in Section 2 the experimental set-up used to perform experimental NACT tests is presented. Section 3 reports the nonlinear ACU experiments for the identification of the driving frequency based on the nonlinear LDR effect and a modified slanted set-up of ACTs relying on the highest signal-to-noise ratio of measured ultrasonic signals. Section 4 shows the result of NACT tests, whilst Section 5 provides a validation of NACT tests with laser Doppler vibrometry scan measurements. Section 6 provides a comparison with NACT experiments and standard Flash thermography and Pulsed Phase thermography. The conclusions of this paper are finally presented in Section 7.

\section{Experimental set-up}

Aircraft engine blades, typically made from titanium and nickel alloys, experience high-cycle fatigue loads due to mechanical vibrations and airflow dynamics in addition to high thermal stresses. Small indentation craters generated by foreign objects can become sites for fatigue micro-crack initiation, thus severely decreasing the lifetime of blades. A $78 \mathrm{~mm}$ Inconel aircraft engine turbine blade with a $1.4 \mathrm{~mm}$ fatigue microflaw in its shroud was selected for testing (Fig. 1). The experimental work included two major elements: nonlinear air coupled ultrasonic tests and thermosonics measurements.

For the nonlinear air-coupled ultrasonic testing, second harmonic was chosen as the nonlinear elastic feature indicating the presence of microcracks. A signal generator (TTi TG5011 Function Generator, $50 \mathrm{MHz}$ ) was used in conjunction with an amplifier (Ciprian US-TXP-3 High Voltage Power Amplifier) and a transmitting point-focused air-coupled transducer (Ultran ACT NCG50-D25-P76, bandwidth 30-70 kHz) with a central frequency of $50 \mathrm{kHz}$. The receiving ACT transducer was, instead, chosen with a central frequency of $100 \mathrm{kHz}$ (ACT NCG100-D25-P76, bandwidth $50-150 \mathrm{kHz}$ ) in order to provide a higher second harmonic elastic response. The received signal was amplified using a pre-amp (Olympus Panametrics-NDT Ultrasonic Preamp $34 \mathrm{~dB}$ ), which was fed into a digital oscilloscope (PicoScope 4424) connected to a personal computer. For the calibration of the air-coupled transmission, ultrasonic signals were also recorded using a contactless 2D laser Doppler vibrometry (LDV) system (Polytec PSV-A-420) with a stand-off distance of $507 \mathrm{~mm}$. Signals received by the LDV system were sampled at $1 \mathrm{MHz}$ with an acquisition window $\tau=2.5 \mathrm{~ms}$ and processed using MATLAB software. Thermography experiments for NACT involved the acquisition of thermal images using a mid-wavelength infrared camera (CEDIP Jade 3 MWIR 3-5 $\mu \mathrm{m}, 320 \times 250$-pixel resolution, average NEDT of $30 \mathrm{mK}$ ) with a fixed frame rate of $25 \mathrm{~Hz}$ and $56 \mathrm{~s}$ recording time (equivalent to 1400 frames). It should be noted that the camera was positioned away from the ACT and was focussed exclusively on the damaged zone without having the ACT included in the video frame. This was to minimise the direct exposure of heat flow from the ACT transducer to the IR camera, which could negatively affect thermal measurements. Additionally, in order to provide a uniform thermal emissivity, the blade was painted black with spray paint. Fig. 2 illustrates the experimental set-up for both nonlinear ultrasonic and thermosonics tests. 


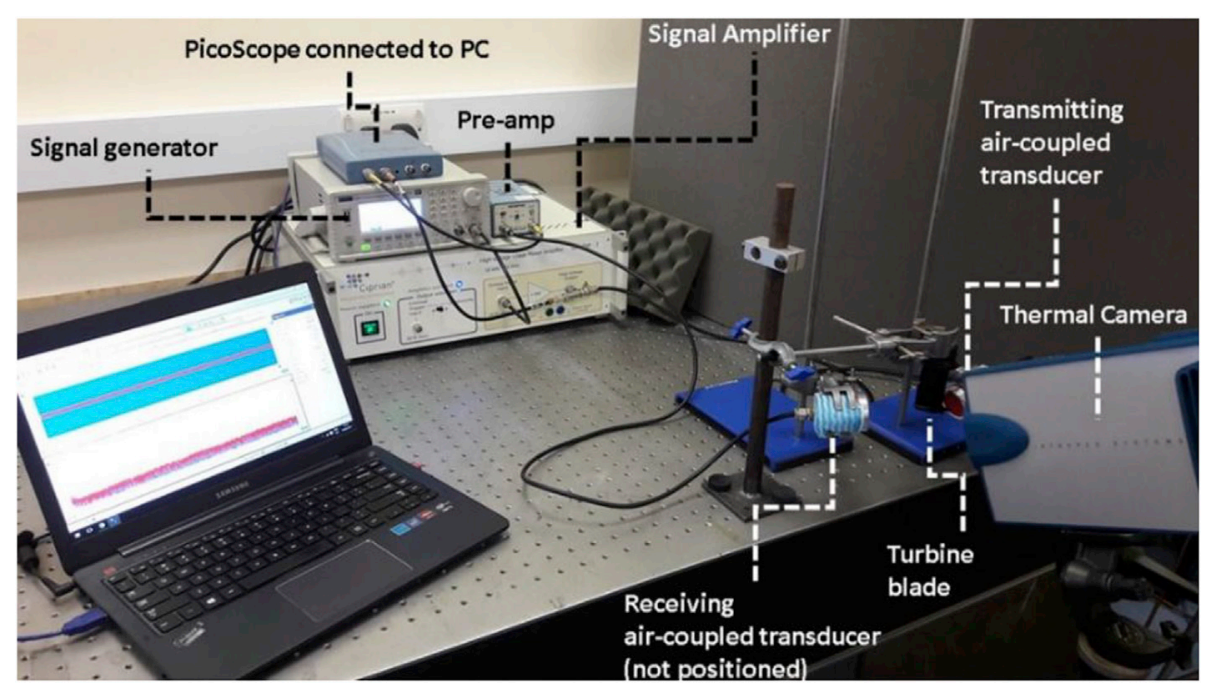

Fig. 2. Experimental set-up of the proposed NACT method.

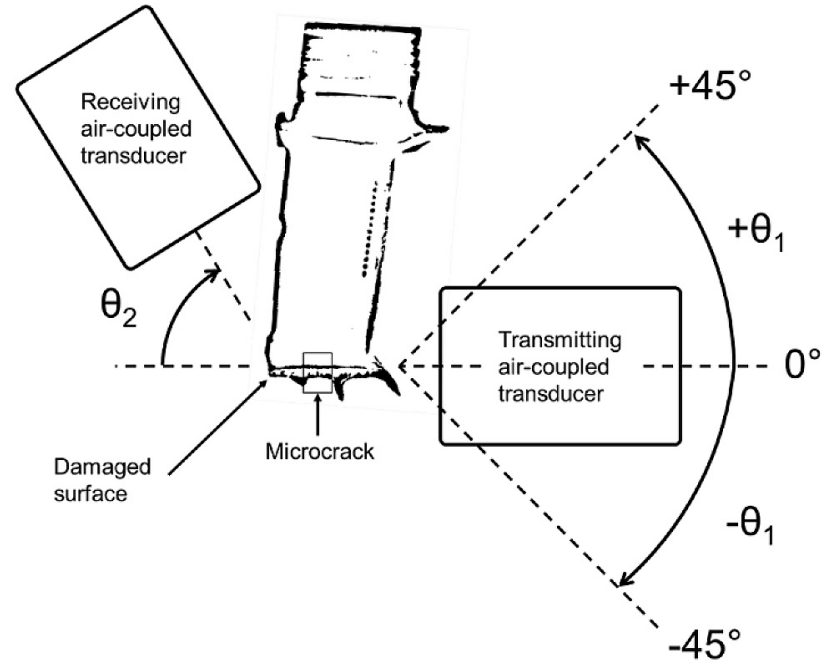

Fig. 3. Illustration of nonlinear air-coupled ultrasonic testing with slanted transmission set-up.

Finally, a thermoscope flash heating system (DM TWI Flash) capable of delivering $2 \mathrm{~kJ} / \mathrm{m}^{2}$ was connected to a pulse generator sending a pulse of $5 \mathrm{~ms}$ width in order to perform both flash and pulsed phase thermography testing.

\section{Nonlinear ACU testing}

The implementation of nonlinear ACU testing required contactless transducers to be set at specific angles. A modified slanted transmission set-up of the air-coupled sensors was here used as it allowed minimising the impact of waves reflected from the blade surface on the received signal. In this set-up, the received signal will be mostly due to waves transmitted through the specimen and from the damaged location (see Fig. 3).

\subsection{Optimisation of the signal-to-noise ratio for ACU transmission}

Initial tests using an LDV system were performed in order to enhance the signal-to-noise ratio (SNR) of measured waveforms during ultrasonic air-coupled transmission. Given the $30-70 \mathrm{kHz}$ frequency bandwidth spectrum of transmitter air-coupled transducers, only fundamental

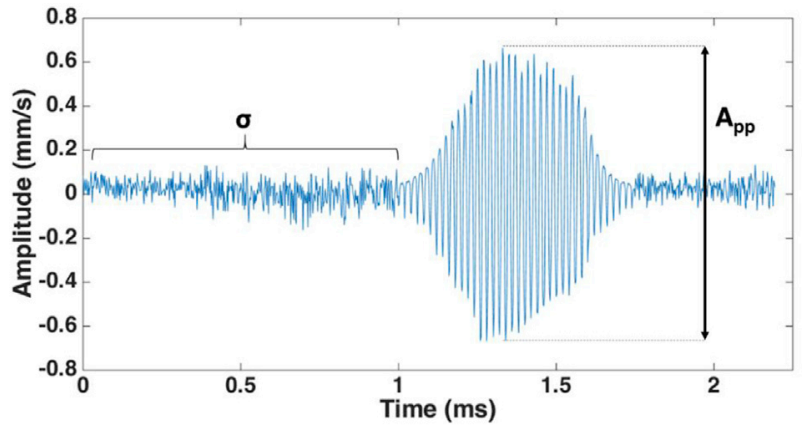

Fig. 4. Sine wave burst signal measured by the laser Doppler vibrometry (LDV) system. In this figure, $A_{p p}$ is the peak-to-peak amplitude of the received signal and $\sigma$ is the standard deviation of noise.

guided wave (Lamb) modes were excited. It is known that, according to the Snell's Law defined as $\theta=\arcsin \left(v_{\text {air }} / v_{s}\right)$, the acoustic energy

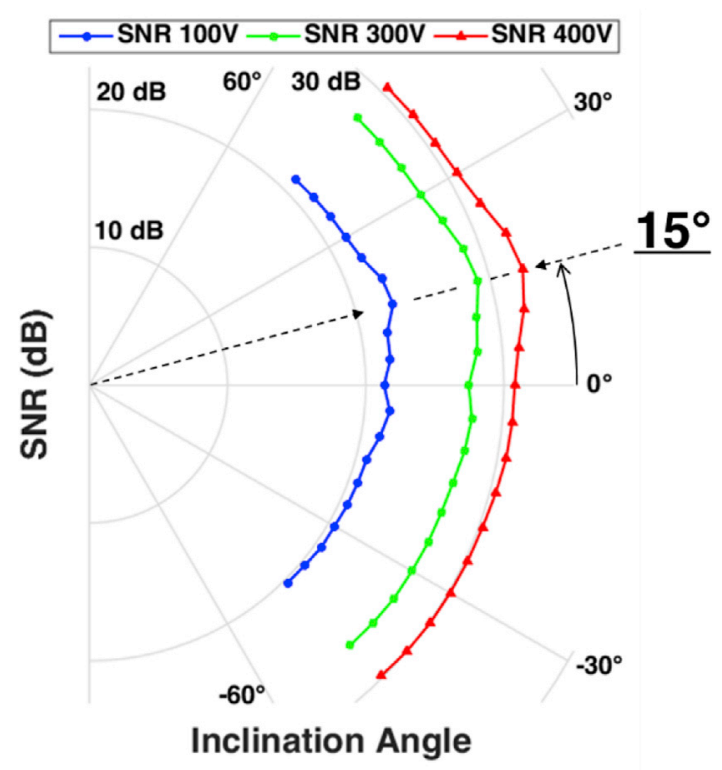

Fig. 5. Variation of SNR as function of the transmitter ACT inclination angle $\theta_{1}$ for three input voltages $A_{0}(100 \mathrm{~V}, 300 \mathrm{~V}$ and $400 \mathrm{~V})$ using the LDV system. 


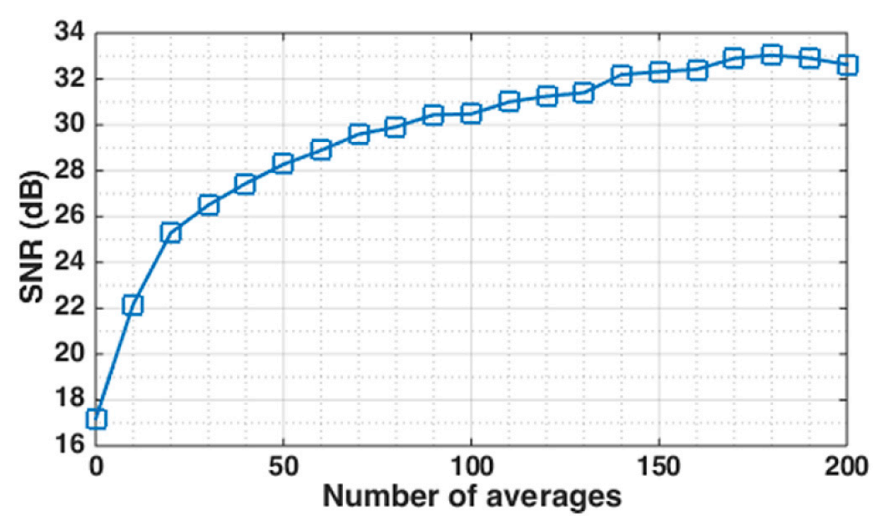

Fig. 6. Variation of SNR as function of time-domain signal averages at $f_{0}=50$ $\mathrm{kHz}, A_{0}=400 \mathrm{~V}$ and $\theta_{1}=15 \pm 0.5$ degrees.

transmitted at the incident angle $\theta$ in the slanted transmission set-up depends on the wave propagation (phase) velocity in both the sample $\left(\mathrm{v}_{s}\right)$ and air $\left(\mathrm{v}_{\text {air }}\right)$. Nevertheless, it is generally difficult to calculate analytically the angle $\theta$ in geometrically complex components such as the turbine micro-damaged blade examined in this paper. Hence, similarly to Dhital and Lee [21], the optimum angle $\theta$ was determined through experimental iterations based on the maximum SNR gain at a given setting. In particular, assuming a lift-off distance of the transmitter ACT of $\sim 15 \mathrm{~mm}$, the generated elastic wave field was recorded by the LDV system at angles $\theta_{1}$ ranging between $\pm 45^{\circ}$, with a limit value of $0^{\circ}$ (planar angle). The highest SNR, defined by Eq. (1),

$S N R=20 \log _{10}\left(\frac{A_{p p}}{\sigma}\right)$

where $A_{\mathrm{pp}}$ is the peak-to-peak amplitude of the received signal and $\sigma$ is the standard deviation of noise, was used to determine the optimal angle $\theta$. For the ultrasonic signal transmission, a 15 cycles long sine wave burst $s(t)=A_{0} H(t) \sin \left(2 \pi f_{0} t\right)$ enclosed in a Hanning window $H(t)$, with $A_{0}$ being the input amplitude and $f_{0}$ the input frequency was used (see Fig. 4).

By setting the driving frequency $f_{0}=50 \mathrm{kHz}$ (i.e. the central frequency of the ACT), Fig. 5 shows the SNR variation for different transmitter inclination angles (with $5^{\circ}$ increments) for three different input voltage amplitudes $\mathrm{A}_{0}$ (i.e. $100 \mathrm{~V}, 300 \mathrm{~V}$ and $400 \mathrm{~V}$ ).

As it can be seen in Fig. 5, SNR variations were more sensitive at lower input voltages, with the highest SNR achieved at $\theta_{1}=15 \pm 0.5$ degrees. This inclination angle $\theta_{1}$ was selected for the nonlinear air-coupled ultrasonic experiments. With regard to the receiving ACT transducer, its position was set on the opposing side of the blade (see Fig. 3) at an angle $\theta_{2}=20 \pm 0.5$ degrees in order to minimise the impact of reflected ultrasonic waves between the sample and the air on the received signal. It should be noted that the difference of five degrees between $\theta_{1}$ and $\theta_{2}$ is likely due to the complex geometry of the blade. Moreover, in order to increase the accuracy of measurements and reduce the noise effects, the time-domain signals were averaged. By setting the excitation frequency $f_{0}=50 \mathrm{kHz}$, the input voltage $A_{0}=400 \mathrm{~V}$ and the inclination angles $\theta_{1}=15^{\circ}$ and $\theta_{2}=20^{\circ}$, non-contact ultrasonic experiments showed that for up to 50 averages the SNR rapidly increased and then converged toward a flat asymptote for higher values (i.e. $\sim 33 \mathrm{~dB}$ at 130 averages, see Fig. 6).

Hence, 130 time-domain averages were chosen for the following experimental contactless nonlinear ultrasonic campaign.

\subsection{LDR frequency-selection using narrowband sweep excitation}

In standard thermosonics tests, the frequency bandwidth is generally chosen from experience of the operator. However, nonlinear ultrasonic
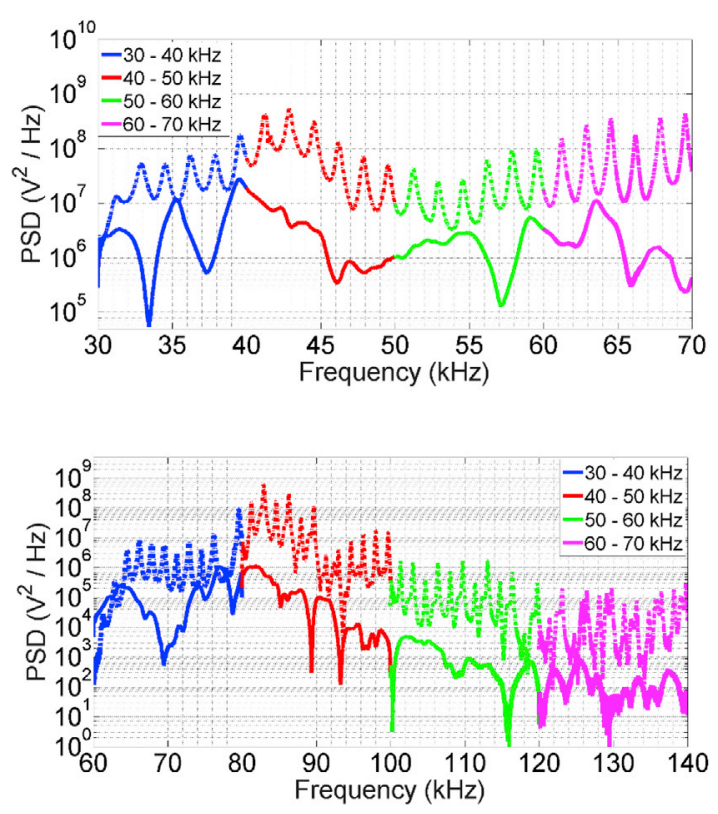

Fig. 7. Power spectral density (PSD) of both material (solid line) and system (dash-dot line) sweep responses in the fundamental (a) and second harmonic (b) regime. Both transmitter and receiver ACTs were used for the ultrasonic testing.

tests from literature (see for instance $[6,27,28]$ ) showed that harmonics of the LDR frequency in damaged metal and composite samples of different geometries with $\mathrm{mm}$-size defects were generated by exciting the material at frequencies ranging between $30 \mathrm{kHz}$ and $70 \mathrm{kHz}$. Hence, such a frequency range was used in this project to excite fatigue micro-cracks with air-coupled transducers. The general mechanism for the generation of higher harmonics, superharmonics, intermodulation LDR effects and frictional heat at damage location is reported both numerically and analytically in Refs. [15] and [16]. With regards to the second order nonlinear elastic effect generated at the LDR location, its expression can be analytically obtained by solving the following nonlinear equation of motion governing the bending of thin homogeneous isotropic plates subject to sinusoidal transverse force:

$D_{11} \frac{\partial^{4} w_{0}}{\partial x^{4}}+2\left(D_{12}+2 D_{66}\right) \frac{\partial^{4} w_{0}}{\partial x^{2} \partial y^{2}}+D_{22} \frac{\partial^{4} w_{0}}{\partial y^{4}}+I_{0} \frac{\partial^{2} w_{0}}{\partial t^{2}}-q+q^{N L}=0$,

where $q$ is the applied sinusoidal transverse force, $q^{N L}$ is the nonlinear force containing the quadratic term associated with the nonlinear LDR, $w_{0}$ is the out-of-plane displacement, $I_{0}$ is the mass moment and $D_{\mathrm{ij}}$ are the components of the bending stiffness matrix. Under the assumption of second order nonlinear approximation, the second order solution of Eq. (2) contains second harmonic term $S_{2}$ and superharmonic term $S_{3}$ associated with the local resonance frequency:

$S_{2}=\frac{\cos \left(2 \pi f_{m n} t\right)-\cos \left(4 \pi f_{0} t\right)}{4 \pi^{2}\left(4 f_{0}^{2}-f_{m n}^{2}\right)}$

$S_{3}=\frac{\cos \left(2 \pi f_{m n} t\right)-\cos \left(4 \pi f_{d} t\right)}{4 \pi^{2}\left(4 f_{d}^{2}-f_{m n}^{2}\right)}$

where $f_{d}$ is the LDR frequency and $f_{m n}$ is one of the resonance modes of the plate. When the frequency of the propagating elastic waves is matched to the LDR frequency of the damaged sample, it implies that $S_{2}$ $=S_{3}$ in Eq. (3) and the second order vibration amplitude of the portion of volume below the defect dramatically increases at $2 f_{0}=2 f_{d}=f_{m n}$. This is due to a local decrease in stiffness for a certain structural mass in the damaged region, which manifests in a particular characteristic frequency $f_{0}=f_{d}$. Although the solution of the nonlinear equation (2) demonstrates 


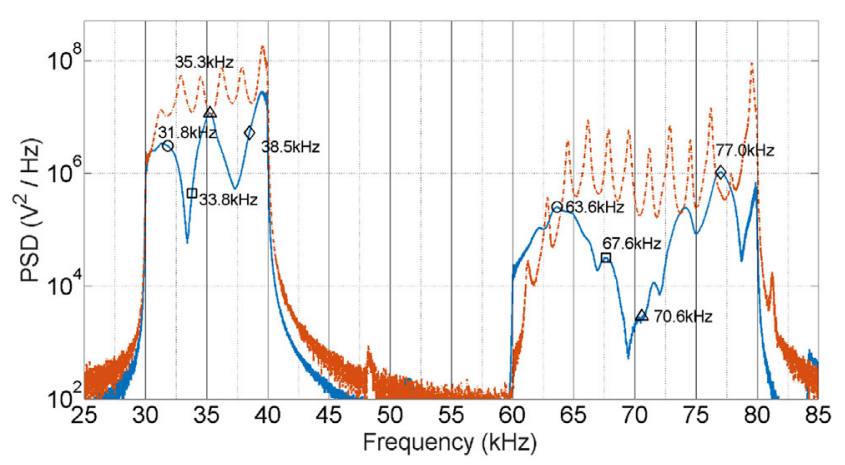

Fig. 8. Power spectral density of both material (blue solid line) and system (red dash-dot line) sweep responses for the frequency range of interest, $30-40 \mathrm{kHz}$. Four input frequencies were selected: $31.8 \mathrm{kHz}$ ("circle" marker), $33.8 \mathrm{kHz}$ ("square" marker), $35.3 \mathrm{kHz}$ ("triangle" marker) and $38.5 \mathrm{kHz}$ ("diamond" marker). Both transmitter and receiver ACTs were used for the ultrasonic testing. (For interpretation of the references to colour in this figure legend, the reader is referred to the Web version of this article.)

the theoretical existence of second harmonic peaks associated with the LDR effect (Eq. (3)), their experimental identification requires narrowband ultrasonic frequency sweeps. It should be also noted that the high input voltages used with ACTs can generate second harmonics due to the acquisition system and not only to the damaged specimen. For this reason, air-coupled ultrasonic tests involved the estimation of the specimen/material response against the transducers one (here named as system response). The latter was defined as the response recorded by the receiving ACT when no specimen was present, i.e. with both ACTs located in a fixed position $100 \mathrm{~mm}$ opposite each other with an inclination angle $\theta$ of $0^{\circ}$. Hence, the driving frequency that showed a high second harmonic material response and a low second harmonic system one was considered as an LDR frequency candidate. Similarly to Fierro et al. [6], four narrowband sweep input signals ranging between $30 \mathrm{kHz}$ and $70 \mathrm{kHz}$ with a frequency span of $10 \mathrm{kHz}$ at the input voltage of $400 \mathrm{~V}$ and a sweep duration time of $10 \mathrm{~s}$ each were generated. Time-domain signals were collected at $1 \mathrm{MHZ}$ sampling rate and a MATLAB code using a fast-Fourier transform (FFT) algorithm was used to compute power spectral density of recorded signals. The results for both the fundamental and second harmonic spectrum range are plotted in Fig. 7.

Different line colours were used to differentiate results for the four sweeps achieved at different frequency ranges. As it can be noticed in Fig. 7(b), the material second harmonic response (solid line) is much higher in the $60-100 \mathrm{kHz}$ range. On the other hand, the system second harmonic response (dash-dot line) is generally higher in the $80-100 \mathrm{kHz}$ range. This means that the second harmonic peaks in the $60-80 \mathrm{kHz}$ range have a higher likelihood to be generated by the micro-crack rather than the acquisition system. Therefore, the driving frequency range of $30-40 \mathrm{kHz}$ was selected for the identification of LDR frequencies. Fig. 8 shows the results for the frequency sweep in the $30-40 \mathrm{kHz}$ range with both the material and the system responses. Two LDR frequency candidates were selected, i.e. $31.8 \mathrm{kHz}$ ("circle" marker) and $38.5 \mathrm{kHz}$ ("diamond" marker), as their second harmonics revealed a peak amplitude in the material response (blue solid line) and a minimum amplitude in the system one (red dash-dot line). It should be noted that even though the frequency $f_{0}=38.5 \mathrm{kHz}$ features a higher material second harmonic response than $31.8 \mathrm{kHz}$, also the system second order response is higher at this frequency. For this reason, it is difficult to establish at this stage of experiments which of these two fundamental frequencies is better for thermosonics testing. Therefore, both driving frequencies were used for the implementation of NACT. In addition, two other frequencies were selected for reference and comparison purposes. Particularly, $f_{0}=33.8 \mathrm{kHz}$ ("square" marker) featured a low fundamental and a second harmonic peak and $f_{0}=35.3 \mathrm{kHz}$ ("triangle" marker) had a large fundamental response, but a very low second harmonic material behaviour.

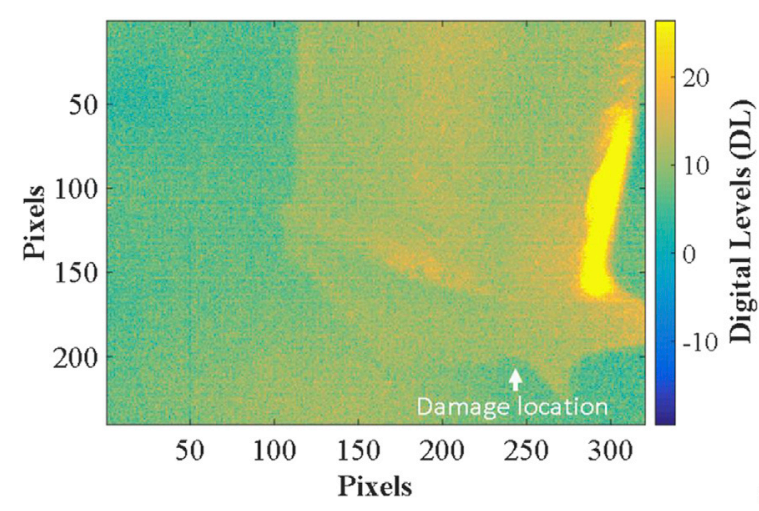

(a)

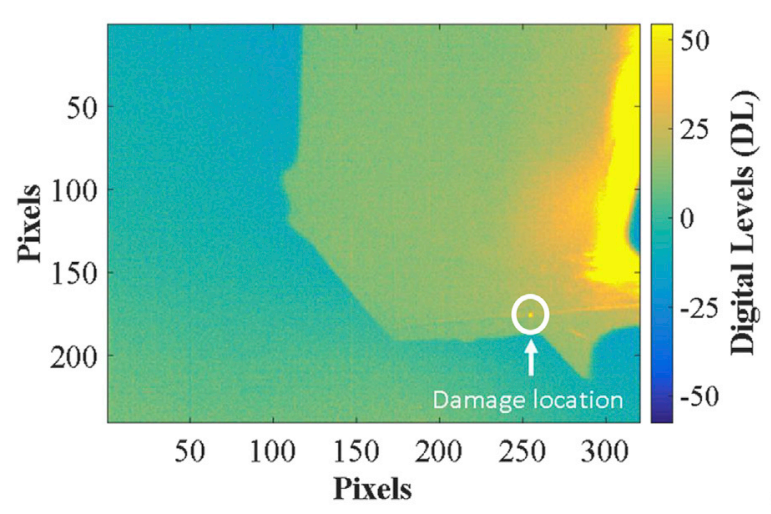

(b)

Fig. 9. NACT thermal amplitude results for continuous excitation frequency at $31.8 \mathrm{kHz}$ with an input voltage of $200 \mathrm{~V}$ (a) and $400 \mathrm{~V}$ (b).

\section{Nonlinear air-coupled thermosonics experiments}

NACT testing was conducted with the thermal camera focussed on the damaged area of the blade and the ACU excitation being triggered with a short delay after starting the thermal image recording. The specimen was excited using a continuous sinusoidal signal at one of the frequencies selected in the previous Section. In accordance with the preliminary results achieved in Section 3.1, Fig. 9 depicts the thermal responses acquired by the IR camera at the excitation frequency $f_{0}=31.8 \mathrm{kHz}$ for two input voltages, $200 \mathrm{~V}$ (a) and $400 \mathrm{~V}$ (b). As expected, the micro-crack became visible only when $400 \mathrm{~V}$ was used, i.e. when sufficient acoustic energy was transmitted into the material in order to trigger the LDR effect and generate enough frictional heat. Thermal images were constructed using the data at the time $t=56 \mathrm{~s}$ and a background subtraction was carried out by selecting a number of pre-excitation frames $P$ and then computing the average for every pixel across these pre-excitation frames. In this case, $P=50$ frames (duration of $2 \mathrm{~s}$ ) were used for the background subtraction.

Fig. 10 shows the NACT thermal amplitude results for the remaining three frequencies previously selected at the input voltage of $400 \mathrm{~V}$. The damage is only detected and localised for the other LDR frequency excited at $f_{0}=38.5 \mathrm{kHz}$. Indeed, there is no conclusive thermal contrast between the micro-crack and its surroundings for driving frequencies at $33.8 \mathrm{kHz}$ and $35.3 \mathrm{kHz}$.

Fig. 11 illustrates the comparison of apparent temperature contrast between the pixel at the damage location and the reference point for NACT tests using the four previously selected driving frequencies. The frequencies with a high second harmonic response (i.e. $31.8 \mathrm{kHz}$ and $38.5 \mathrm{kHz}$ ) resulted in a visible DL contrast. On the contrary, the apparent temperature difference for the other two frequencies (i.e. $33.8 \mathrm{kHz}$ and $35.3 \mathrm{kHz}$ ) was negligible.

Furthermore, the time domain data were used to plot phase images. The procedure to extract the phase from the thermal signals is based on 


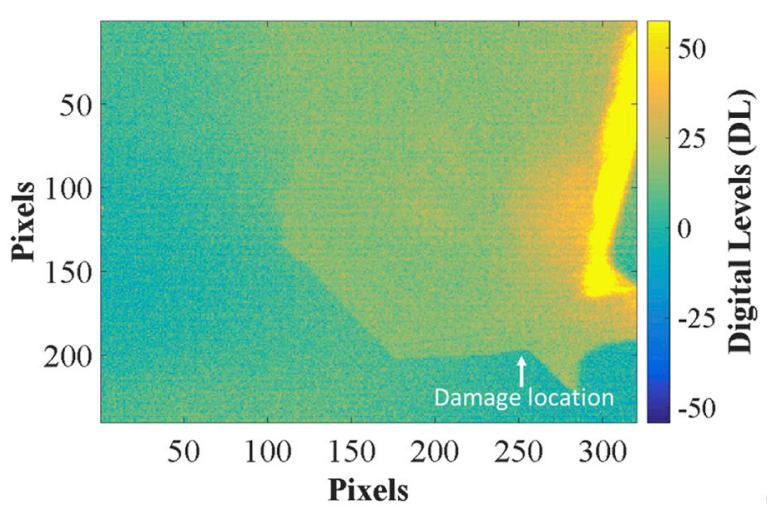

(a)

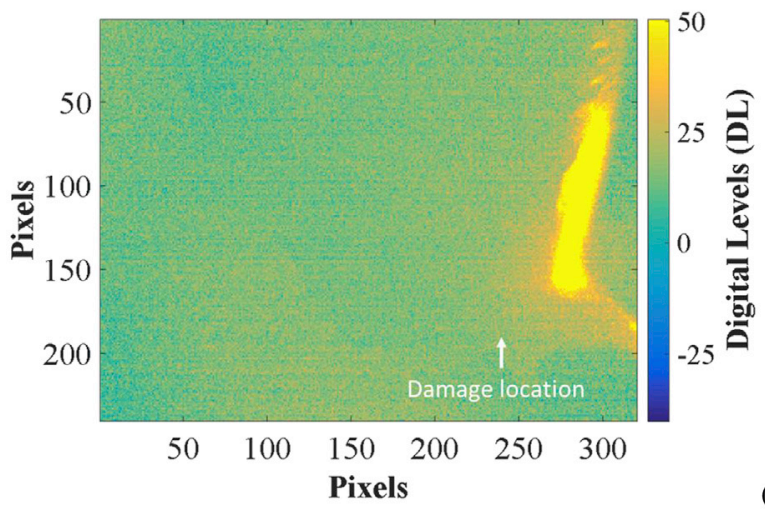

(b)

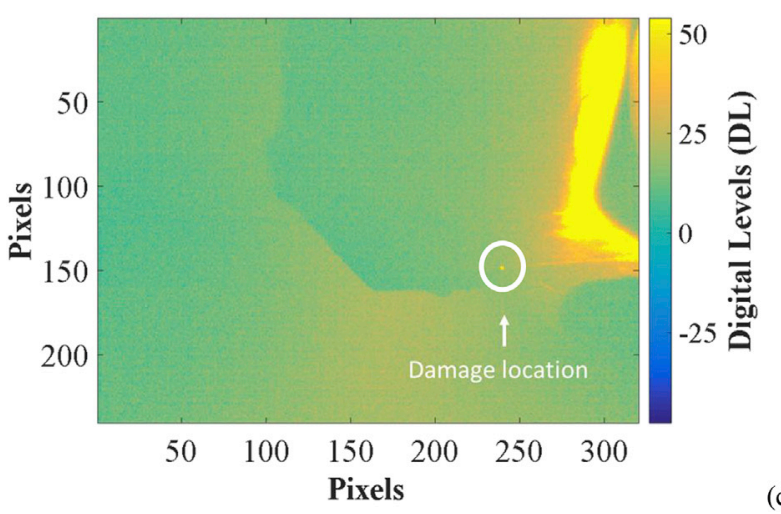

(c)

Fig. 10. NACT thermal amplitude results for continuous excitation frequency at $33.8 \mathrm{kHz}$ (a), $35.3 \mathrm{kHz}$ (b) and $38.5 \mathrm{kHz}$ (c) with an input voltage of $400 \mathrm{~V}$.

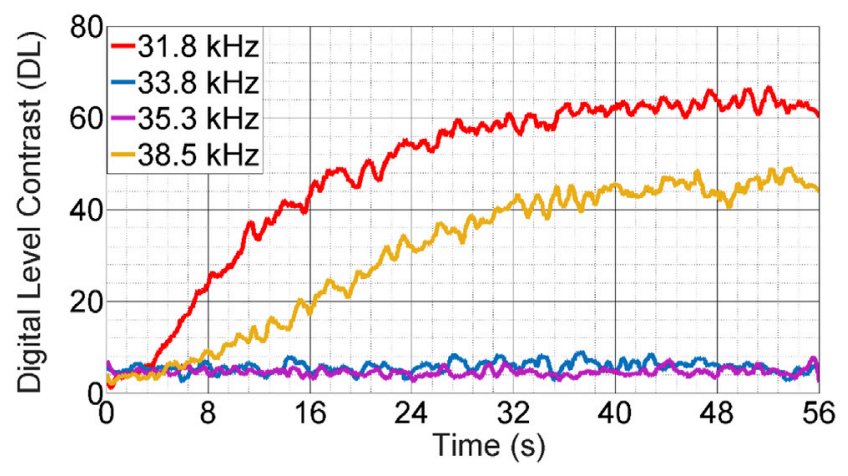

Fig. 11. Comparison of NACT thermal amplitude contrast variation for the four investigated driving frequencies, i.e. $31.8 \mathrm{kHz}, 33.8 \mathrm{kHz}, 35.3 \mathrm{kHz}$ and $38.5 \mathrm{kHz}$.

the Fast Fourier Transform $\widehat{s}(f)=\operatorname{Re}(f)+\operatorname{Im}(f)$, where $\operatorname{Re}(f)$ and $\operatorname{Im}(f)$

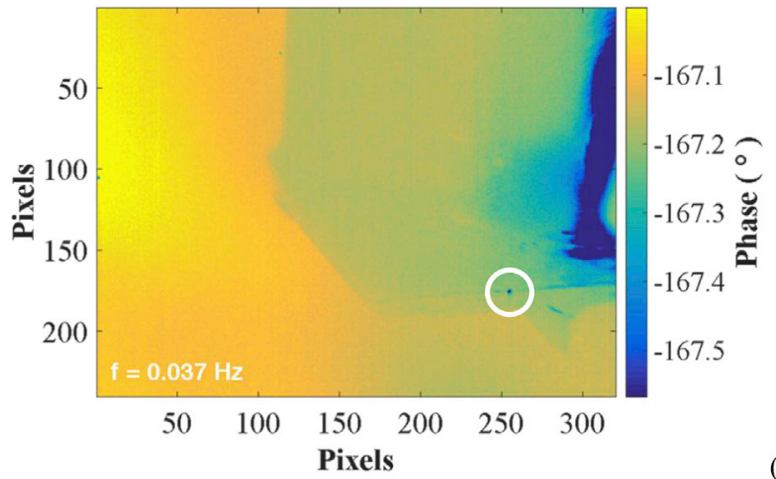

(a)

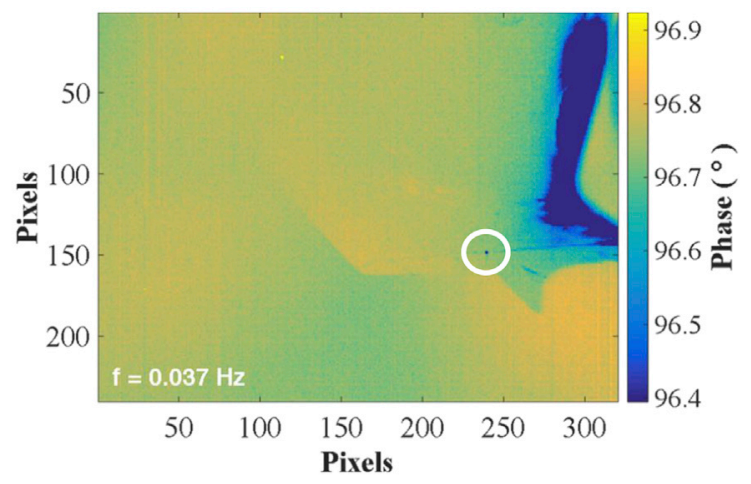

(b)

Fig. 12. NACT phase images obtained at the driving frequency $f_{0}=31.8 \mathrm{kHz}$ (a) and $f_{0}=38.5 \mathrm{kHz}(\mathrm{b})$.

are the real and imaginary parts of the FFT, $\widehat{s}(f)$, of apparent temperature data. Hence, the phase image for every pixel at certain thermal frequencies was obtained as the arctangent of the ratio between the imaginary and the real part of the FFT [29]. Fig. 12 shows that for the case of sinusoidal excitation at $f_{0}=31.8 \mathrm{kHz}$, the phase image revealed the presence of damage at the thermal frequency $f=0.037 \mathrm{~Hz}$. The same thermal frequency was also used to retrieve the damage location at the ultrasonic driving frequency $f_{0}=38.5 \mathrm{kHz}$.

\subsection{Enhancement of NACT thermal amplitude images}

Although the fatigue micro-damage was successfully detected and localised using the proposed NACT technique [see Figs. 9-12], both anisotropic heating and thermal noise effects were clearly visible in the thermal images as a result of the high input voltage applied to the ACT. In order to mitigate these detrimental effects and enhance the quality of thermal results, a smoothing filter and a secondary background subtraction using morphological opening were implemented. Morphological opening is an operation that is widely used in computer vision technology to transform the image by computing a new value for every pixel based on the comparison with a cluster of neighbouring pixels [30]. Morphological opening can be used to estimate the background of an image affected by non-uniform illumination as in standard photography. Since the anisotropic heating is here analogous to non-uniform illumination, morphological opening was used to extract the image background affected by non-uniform heating and then subtract it from the original image. Due to the low spatial resolution of the thermal camera, the obtained image data matrices were resized by a factor of five in order to facilitate image filtering. Morphological opening was combined with a Savitzky-Golay smoothing filter. A Savitzky-Golay filter [31] is a polynomial fitting digital filter that is usually used for smoothing wideband noisy signals. However, it is also applicable to image smoothing, although this area has been explored to a lesser extent [32] In a Savitzky-Golay filter, a polynomial function is fit to a signal with the 


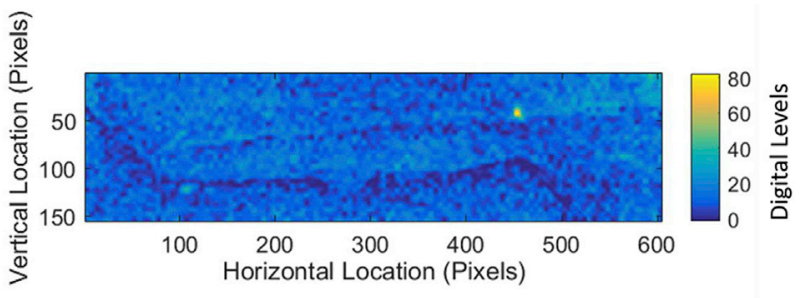

Fig. 13. Thermal amplitudes images of the fatigue micro-crack $5 \times$ enlarged and filtered using morphological opening and a Savitzky-Golay filter at the input frequency of $31.8 \mathrm{kHz}$.

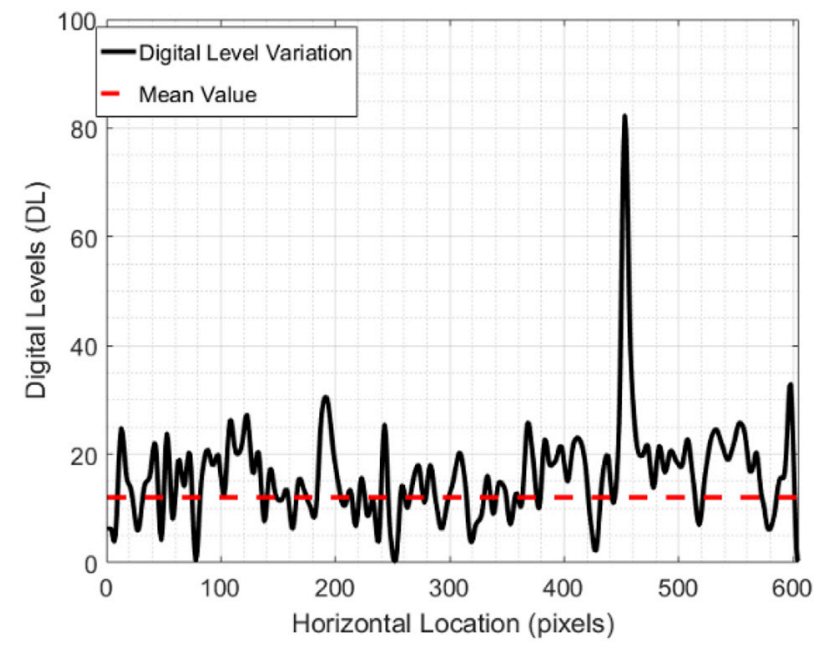

Fig. 14. Digital level variation versus mean value of digital levels across the horizontal line surrounding the micro-crack using morphological opening and smooth filtering.

least-squares method and the original signal values are replaced with filtered values. Considering a 1D noisy thermal signal $s(n)$ with a total number of samples equal to $N(n=1, \ldots, N)$ and a Savitzky-Golay window of length $2 M+1$ (from $-M$ to $M$ ), the window is moved along the length of the signal, approximating the central value at each step [31]. This approximation is the best least-squares fit to a polynomial $p(\mathrm{k})$ of a specific even degree $k$, where

$p(k)=\sum_{r=0}^{k} a_{r} x^{r}=a_{0} x^{0}+a_{1} x^{1}+a_{2} x^{2}+\cdots+a_{k} x^{k}$.

The mean-squared approximation error $\xi_{k}$ is calculated as follows:

$\xi_{k}=\sum_{m=-M}^{M}[p(k)-s(m)]^{2}=\sum_{m=-M}^{M}\left[\sum_{r=0}^{k} a_{r} x_{m}^{r}-s(m)\right]^{2}$.

The values for the $p(k)$ polynomial fitting coefficients were computed by using the signal data in a vector format and then constructing a matrix equation. For the thermal image processing, the algorithm simply operates by smoothing the data from subsequent columns of a $2 \mathrm{D}$ image matrix. It should also be noted that more advanced techniques could be used by deriving a two-dimensional Savitzky-Golay filter kernel [32] Fig. 13 shows the post-processed thermal image focusing on the damaged blade shroud area. In particular, it shows the filtered thermal plot enhanced by a combination of secondary background subtraction using morphological opening and the Savitzky-Golay filter at the input frequency of $31.8 \mathrm{kHz}$.

As it can be seen in Fig. 13, the secondary background subtraction via morphological opening with a smoothing filter enhanced detectability and localisation of the crack. Indeed, Fig. 14 illustrates thermal variations

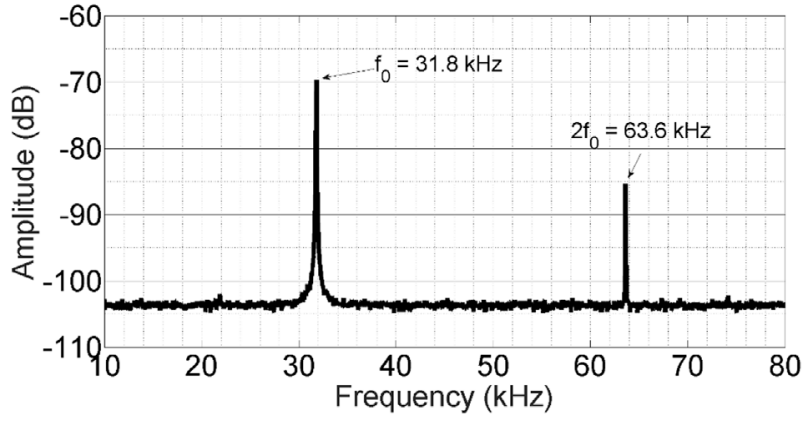

Fig. 15. Average LDV scan spectrum for continuous excitation using a driving frequency at $31.8 \mathrm{kHz}$ and an input voltage at $400 \mathrm{~V}$.
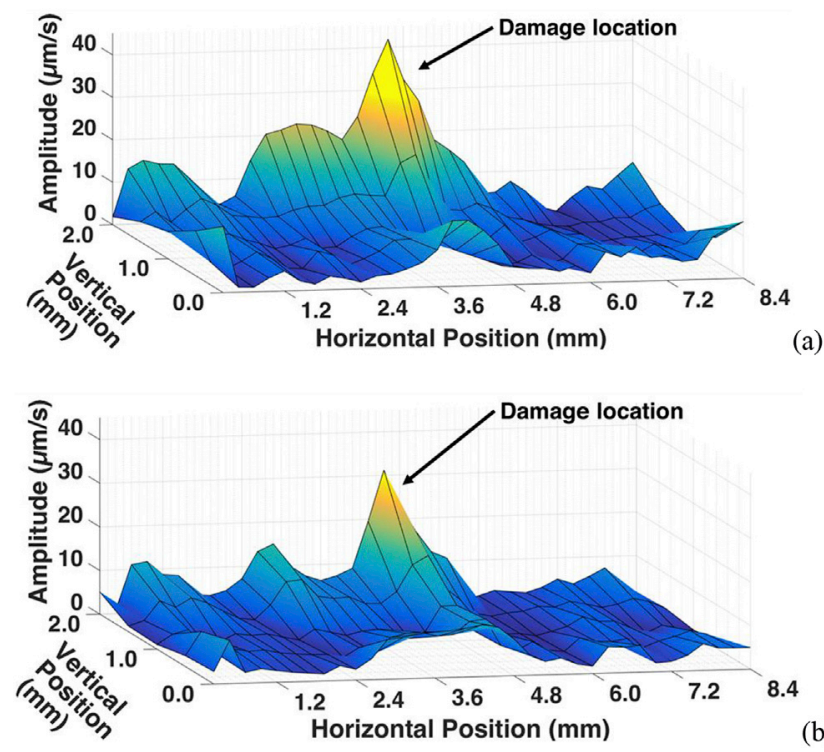

(b)

Fig. 16. Laser Doppler vibrometry second harmonic response for the input frequency of $31.8 \mathrm{kHz}$ (a) and $38.5 \mathrm{kHz}$ (b) at $400 \mathrm{~V}$.

across the horizontal line of the thermal amplitude image in Fig. 13 (black continuous line) using morphological opening and smoothing filter. From this Figure it can be seen that the maximum digital level is $\sim 80$ at the micro-crack location. The dashed red line in Fig. 14 is the mean value of digital levels ( $\sim 10$ digital levels), which reveals that the thermal contrast between the maximum digital level at damage location and the mean value is $\sim 70$ digital levels. Such a value is higher than the $\sim 65$ digital levels without morphological opening and filtering (see the maximum thermal amplitude contrast at $31.8 \mathrm{kHz}$ in Fig. 11).

\section{Validation of NACT using a Laser Doppler Vibrometer}

Following the same ACU set-up reported in Section 3.1, the 2D LDV scanning system was now used to validate the NACT imaging results and demonstrate that the thermal contrast between the micro-crack and its surroundings was the result of frictional heating generated exclusively at the LDR (micro-crack) location. The scanned region on the blade was an area around the damage of $2.0 \mathrm{~mm} \times 8.4 \mathrm{~mm}$ using 175 scanning points in a $5 \times 35$ grid. Fig. 15 shows the generation of second harmonic $2 f_{0}=63.6 \mathrm{kHz}$ from the measured average spectrum using the LDV scan at $f_{0}=31.8 \mathrm{kHz}$ and $A_{0}=400 \mathrm{~V}$.

Fig. 16 depicts the second harmonic response from the LDV scan for the two LDR driving frequencies $(31.8 \mathrm{kHz}$ and $38.5 \mathrm{kHz})$. As a first consideration, both images show that the two driving frequencies (and their associated eigenmodes) are inherently connected to the spatial 


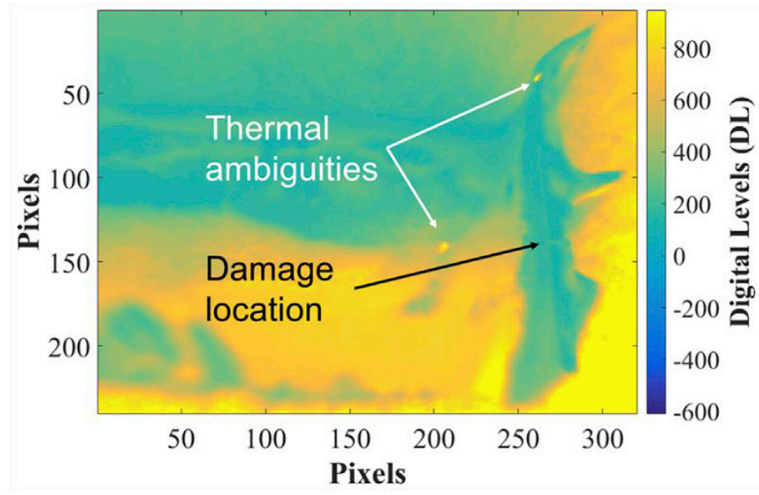

(a)

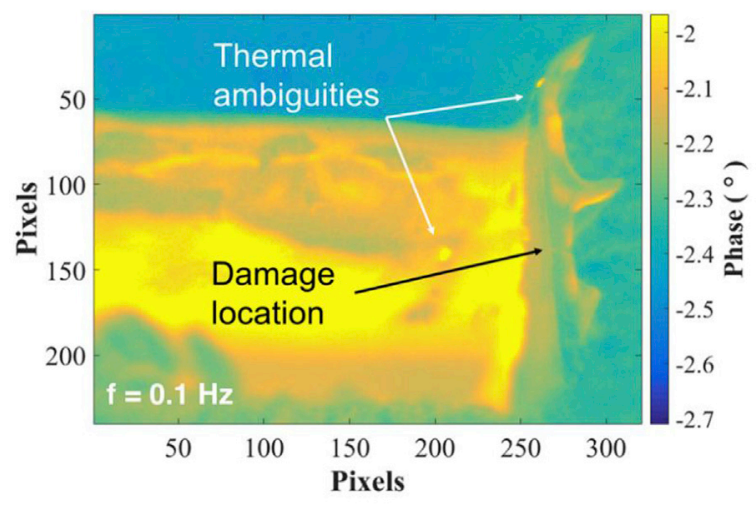

(b)

Fig. 17. Flash thermography (a) and pulsed phase thermography (b) thermal images of the damaged blade.

location on the blade shroud surface as the peak of the vibrational amplitude (i.e. the velocity measured by the LDV system) occurs exactly at that particular location. This indicates that the excitation of the microdamaged blade at those input frequencies resulted in maximum out-ofplane velocity at the crack interfaces. Secondly, the vibrational amplitude peak at the damage location is higher for $31.8 \mathrm{kHz}$ than for $38.5 \mathrm{kHz}$. This result agrees with the NACT thermal plots in Fig. 11. It is also worth noting that the ultrasonic testing did not highlight this relation (see Fig. 8), as it had appeared that $38.5 \mathrm{kHz}$ would be a better LDR frequency since it featured a higher second harmonic material response.

\section{Comparison with standard flash thermography and pulsed phase thermography}

As a final element of this study, NACT was compared against standard flash thermography and pulsed phase thermography [33]. In order to accurately compare optically stimulated thermography and the proposed NACT technique, the test settings for optical thermographic methods were set to resemble those used in NACT. Particularly, thermal images of the damaged area were recorded over the period of $56 \mathrm{~s}$ with the flash being triggered shortly after the start of the recording (approximately $2 \mathrm{~s}$ delay). Both amplitude (at $t=2.25 \mathrm{~s}$ ) and phase (at $f=0.1 \mathrm{~Hz}$ ) images in Fig. 17 showed that optical thermography was not successful in retrieving the damage location and that thermal ambiguities were present in both apparent temperature and phase plots. These effects were likely to be due to surface imperfections caused by non-uniform heating and uneven black spray paint distribution.

Hence, thermal results in Fig. 17 revealed that flash thermography was not suitable for detecting micro-cracks in small specimens of complex geometry. Conversely, NACT successfully detected and localised fatigue micro-damage, so it can be considered as a valid alternative to traditional optical thermography for micro-flaws identification in geometrically complex structures such as engine turbine blades.

\section{Conclusions}

This paper presented a novel nonlinear air-coupled thermosonics (NACT) technique in which contactless nonlinear ultrasonic experiments and thermography were combined for a rapid and accurate detection and localisation of a fatigue micro-crack in an engine turbine blade. Initial ultrasonic calibration tests with a laser vibrometry system showed that an incident angle of $15^{\circ}$ for the transmitter air-coupled transducer was necessary to achieve the highest signal-to-noise ratio of measured waveforms. Nonlinear ultrasonic testing with air-coupled transducers and narrowband frequency sweeps were then performed in order to identify local defect resonant (LDR) frequencies based on a high second harmonic response. The continuous excitation of these LDR frequencies at the input voltage of $400 \mathrm{~V}$ allowed compensating for signal losses of air-coupled transducers through the actuation of large vibration amplitudes at the micro-crack location. An infrared camera was used to perform NACT experiments by acquiring the material thermal response generated by the frictional heat, thus successfully detecting and localising the fatigue micro-crack. Moreover, an image processing method based on a combination of morphological opening and a Savitzky-Golay smoothing filter was used to enhance the quality of thermal images affected by anisotropic heating and thermal noise effects. NACT results were confirmed and validated with laser Doppler scan measurements. Moreover, the NACT method was compared with traditional optical thermographic methods such as flash thermography and pulsed phase thermography. Thermal results showed that NACT was the only thermographic technique capable of identifying fatigue damage in the blade shroud. Hence, the proposed nonlinear air-coupled thermosonics method can be recognised as a promising solution for quality control and nondestructive inspection of aerospace components with complex geometries and small dimensions.

\section{Acknowledgments}

FC and MM acknowledge the EPSRC "EP/N016386/1" and Horizon 2020 "EXTREME" projects to support this research work.

\section{References}

[1] Bates D, Smith G, Lu D, Hewitt J. Rapid thermal non-destructive testing of aircraft components. Compos B Eng 2000;31(3):175-85.

[2] Ciampa F, Mahmoodi P, Pinto F, Meo M. Recent advances of active infrared thermography for non-destructive testing of aerospace components. Sensors 2018; 18(2):609.

[3] Barden TJ, Almond DP, Pickering SG, Morbidini M, Cawley P. Detection of impact damage in CFRP composites by thermosonics. Nondestr Test Eval 2007;22(2-3): 71-82.

[4] Bolu G, Gachagan A, Pierce G, Harvey G. Reliable thermosonic inspection of aero engine turbine blades. Insigh Non Destr. Test. Cond. Monit 2010;52(9):488-93.

[5] Solodov I, Rahammer M, Derusova D, Busse G. Highly-efficient and noncontact vibro-thermography via local defect resonance. Quant. InfraRed. Thermogr. J. 2015;12(1):98-111.

[6] Fierro GPM, Ginzburg D, Ciampa F, Meo M. Nonlinear ultrasonic stimulated thermography for damage assessment in isotropic fatigued structures. J Sound Vib 2017;404:102-15.

[7] Dionysopoulos D, Fierro GPM, Meo M, Ciampa F. Imaging of barely visible impact damage on a composite panel using nonlinear wave modulation thermography. NDT \& E International 2018;95:9-16.

[8] Solodov I. Ultrasonics of non-linear contacts: propagation, reflection and NDEapplications. Ultrasonics 1998;36(1-5):383-90.

[9] Van Den Abeele KA, Johnson PA, Sutin A. Nonlinear elastic wave spectroscopy (NEWS) techniques to discern material damage, part I: nonlinear wave modulation spectroscopy (NWMS). Res Nondestr Eval 2000;12(1):17-30.

[10] Meo M, Polimeno U, Zumpano G. Detecting damage in composite material using nonlinear elastic wave spectroscopy methods. Appl Compos Mater 2008;15(3): 115-26.

[11] Ciampa F, Scarselli G, Pickering S, Meo M. Nonlinear elastic wave tomography for the imaging of corrosion damage. Ultrasonics 2015;62:147-55.

[12] Ciampa F, Pickering SG, Scarselli G, Meo M. Nonlinear imaging of damage in composite structures using sparse ultrasonic sensor arrays. Struct Contr Health Monit 2017;24(5).

[13] Fierro GPM, Meo M. Residual fatigue life estimation using a nonlinear ultrasound modulation method. Smart Mater Struct 2015;24(2):025040. 
[14] Solodov I, Bai J, Bekgulyan S, Busse G. A local defect resonance to enhance acoustic wave-defect interaction in ultrasonic nondestructive evaluation. Appl Phys Lett 2011;99(21):211911.

[15] Ciampa F, Scarselli G, Meo M. On the generation of nonlinear damage resonance intermodulation for elastic wave spectroscopy. J Acoust Soc Am 2017;141(4): 2364-74.

[16] Fierro GPM, Ginzburg D, Ciampa F, Meo M. Imaging of barely visible impact damage on a complex composite stiffened panel using a nonlinear ultrasound stimulated thermography approach. J Nondestr Eval 2017;36(4):69.

[17] De Angelis G, Meo M, Almond DP, Pickering SG, Angioni SL. A new technique to detect defect size and depth in composite structures using digital shearography and unconstrained optimization. NDT E Int 2012;45(1):91-6.

[18] Delrue S, Van Den Abeele K, Blomme E, Deveugele J, Lust P, Matar OB. Twodimensional simulation of the single-sided air-coupled ultrasonic pitch-catch technique for non-destructive testing. Ultrasonics 2010;50(2):188-96.

[19] Chimenti D. Review of air-coupled ultrasonic materials characterization. Ultrasonics 2014;54(7):1804-16.

[20] Solodov I, Busse G. Nonlinear air-coupled emission: the signature to reveal and image microdamage in solid materials. Appl Phys Lett 2007;91(25):251910.

[21] Dhital D, Lee J. A fully non-contact ultrasonic propagation imaging system for closed surface crack evaluation. Exp Mech 2012;52(8):1111-22.

[22] Lim HJ, Song B, Park B, Sohn H. Noncontact fatigue crack visualization using nonlinear ultrasonic modulation. NDT E Int 2015;73:8-14.

[23] Thiele S, Kim JY, Qu J, Jacobs LJ. Air-coupled detection of nonlinear Rayleigh surface waves to assess material nonlinearity. Ultrasonics 2014;54(6):1470-5.
[24] Zalameda JN, Winfree WP, Yost WT. Air coupled acoustic thermography (ACAT) inspection technique. In: AIP conference proceedings; 2008 [AIP].

[25] Gan TH, Hutchins DA, Billson DR, Schindel DW. The use of broadband acoustic transducers and pulse-compression techniques for air-coupled ultrasonic imaging. Ultrasonics 2001;39(3):181-94.

[26] Solodov I, Rahammer M, Gulnizkij N, Kreutzbruck M. Noncontact sonic NDE and defect imaging via local defect resonance. J Nondestr Eval 2016;35(3):48.

[27] Scarselli G, Ciampa F, Nicassio F, Meo M. Non-linear methods based on ultrasonic waves to analyse disbonds in single lap joints. Proc IME C J Mech Eng Sci 2017. 0954406217704222.

[28] Hettler J, Tabatabaeipour M, Delrue S, Van Den Abeele K. Detection and characterization of local defect resonances arising from delaminations and flat bottom holes. J Nondestr Eval 2017;36(1):2.

[29] Rabiner LR, Gold B. Theory and application of digital signal processing777. Englewood Cliffs, NJ: Prentice-Hall, Inc.; 1975. p. 1975.

[30] Singh P, Garg A. Non uniform background removal using morphology-based structuring element for particle analysis. Int J Comput Appl 2011;33(6):11-6.

[31] Savitzky A, Golay MJ. Smoothing and differentiation of data by simplified least squares procedures. Anal Chem 1964;36(8):1627-39.

[32] Kim JH, Jeung GW, Lee JW, Kim KS. Performance evaluation of a two-dimensional savitzky-golay filter for image smoothing applications, in electronics, communications and networks V. Springer; 2016. p. 309-16.

[33] Balageas D, Maldague X, Burleigh D, Vavilov VP, Oswald-Tranta B, Roche JM, Carlomagno GM. Thermal (IR) and other NDT techniques for improved material inspection. J Nondestr Eval 2016;35(1):18. 